\title{
Valoración del alumnado de los experimentos virtuales frente a los experimentos tradicionales
}

Juan José Serrano-Pérez ${ }^{\mathrm{a}^{*}}$, Lorena González-García ${ }^{b^{*}}$, Alida Taberner-Cortés ${ }^{\mathrm{a}}$, Nicla Flacco ${ }^{\text {a }}$ Isabel García-Arnandis ${ }^{\text {a }}$, Gonzalo Pérez-López .

a Universidad Europea de Valencia, Departamento de Ciencias Biomédicas, c/General Elío, 8, 46010 Valencia. ' Universidad Europea de Valencia, Departamento de Psicología, c/General Elío, 8, 46010 Valencia.

*juanjose.serrano@universidadeuropea.es, lorena.gonzalez@universidadeuropea.es

\begin{abstract}
Traditional laboratory experiments are critical to the learning process across all areas and levels of study in science subjects. However, along with hands-on experiments, virtual labs have received considerable attention over the past several years due to the popularisation of ICT tools in Education, which may help teachers consider multiple learning styles to pursue meaningful learning. In this regard, studies concerning pupils' experiences with both approaches are needed.

The main aim of this study is to analyse the impact of the use of both virtual and hands-on labs in basic science subjects (BSS) in Health Sciences university degrees. For this reason, we have analysed different variables: general assessment, level of satisfaction, increase in motivation and increase in academic performance concerning BSS, as well as gender differences in the perception of both methodologies.
\end{abstract}

A total of 129 undergraduate students from one Spanish university (degree in Dentistry) participated in the study. The results point out to statistically significant differences in favour of traditional labs in all the considered parameters.

In conclusion, the results point out the importance of carrying out hands-on experiments to boost students' motivation and performance.

Keywords: laboratory, ICT, simulation, interactivity, active learning.

\footnotetext{
Resumen

Las prácticas de laboratorio tradicionales juegan un papel protagonista en la enseñanza de las ciencias en todas las áreas y niveles. Sin embargo, en los últimos años la popularidad de los laboratorios virtuales ha aumentado significativamente, debido a que la aplicación de las herramientas TIC en educación permite atender adecuadamente a la diversidad del alumnado y fomentar el aprendizaje activo. En este sentido, resulta necesario estudiar las experiencias del alumnado con ambos enfoques.
} 
El principal objetivo de este estudio es analizar el impacto de los laboratorios virtuales y tradicionales en asignaturas de Ciencias Básicas (CCBB) en un grado de Ciencias de la Salud en el contexto universitario. Para ello se ha estudiado la valoración del alumnado, su grado de satisfacción general, su motivación y su rendimiento en las asignaturas de CCBB, así como la existencia de posibles diferencias de género.

Los participantes del estudio fueron 129 estudiantes del grado en Odontología de una universidad española. Los resultados indican que existen diferencias significativas a favor de las prácticas tradicionales en todas las variables estudiadas.

Como conclusión, se resalta la importancia de fomentar las prácticas tradicionales en grados de Salud para potenciar la motivación y el rendimiento del alumnado.

Palabras clave: laboratorio, TIC, simulación, interactividad, aprendizaje activo.

\section{Introducción}

En la enseñanza del siglo XXI, las TIC (Tecnologías de la Información y Comunicación) aplicadas a la educación (es decir, las TAC, Tecnologías del Aprendizaje y del Conocimiento) ocupan un lugar protagonista. Con su uso, se pretende fomentar una mayor implicación por parte del alumnado, lo cual constituye la base del constructivismo (Coll, 2007; Hyslop-Margison, 2007; Phillips, 1995; Pozo, 2006; Spencer, 1999), que fomenta el aprendizaje activo y significativo (Freeman, 2014; Michael, 2006; Pinto, 2008; Prince, 2004). Esta corriente es especialmente importante en las orientaciones metodológicas que los expertos proponen a los docentes de las asignaturas de Ciencias (Pinto, 2008; Pozo, 2006; Rocard, 2007; Sanmartí, 2009), fomentando la comprensión frente a la mera memorización, esto es, desarrollando habilidades cognitivas de orden superior, de acuerdo con la taxonomía de Bloom de los objetivos didácticos (Cook, 2013; Krathwohl, 2002).

Con el auge de Internet y las TIC (Livingstone, 2012), no sólo la enseñanza presencial tradicional está siendo complementada con otras modalidades como e-learning, b-learning y m-learning (Franco, 2013), sino que se ha asentado en las modernas corrientes pedagógicas un cambio de paradigma en el proceso de enseñanza-aprendizaje: pasar de un sistema educativo basado en la enseñanza a otro distinto basado en el aprendizaje, alejándose de la perspectiva tradicional según la cual "contar" algo en clase equivalía a “enseñar”. En otras palabras, la típica clase magistral se está convirtiendo en un concepto anacrónico.

En definitiva, se trata de fomentar el aprendizaje activo mediante el uso de estrategias metodológicas centradas en el estudiante (Coll, 2007), dando cada vez menos importancia a las clases magistrales (Mazur, 2009) y fomentando el uso de actividades que constituyan un

(cc) BY-NC-ND 2018, Universitat Politècnica de València

Congreso IN-RED (2018) 
reto abordable para los estudiantes, teniendo en cuenta sus competencias actuales y su potencial, permitiendo que desarrollen sus capacidades dentro de la zona de desarrollo próximo (Coll, 2007; Pozo, 2006; Smagorinsky, 1995; Sanmartí, 2009), que es la distancia entre el nivel de desarrollo efectivo del alumno (aquello que es capaz de hacer por sí mismo) y su nivel de desarrollo potencial (aquello que sería capaz de hacer con la ayuda del docente o de un compañero más capaz). Este enfoque es crucial para incentivar la competencia de "aprender a aprender". Básicamente, cuanto más implicado está un estudiante en una actividad dada, más y mejor aprenderá, tal y como pone de manifiesto el cono de la experiencia de Dale (Herreid, 2012; Sprawls, 2008).

La realización de prácticas de laboratorio en las ciencias experimentales es esencial para motivar al alumnado y para la adquisición de contenidos procedimentales importantes en su formación académica y en su futuro ejercicio profesional (ACS, 2013; Domin, 1999; Elliott, 2008; Hofstein, 2003; NSTA, 2013). Sin duda, las prácticas de laboratorio constituyen una herramienta fundamental en las carreras científicas. Sin embargo, como alternativa y complemento a las prácticas de laboratorio, el uso de aplicaciones informáticas de simulación está actualmente en boga.

Los laboratorios virtuales son plataformas interactivas que se adaptan a diferentes estilos y ritmos de aprendizaje, y que suelen estár disponibles gratuitamente para todas las Ciencias Básicas, como Física, Química y Biología. Su uso presenta muchas ventajas (Bortnik, 2017; Chan, 2009; Climent-Bellido, 2003; Cobb, 2009; De Jong, 2013; Franco, 2013; García Armada, 2016; Heradio, 2016; Jensen, 2013): fomentan el trabajo en equipo, son motivantes, implican menos costes que los laboratorios tradicionales, fomentan la creatividad y un aprendizaje más autónomo, se pueden repetir las experiencias tantas veces como sea necesario, disminuyen el miedo al error y al fracaso por parte del discente, facilitan la visualización de procesos y conceptos abstractos, y permiten al docente llevar a cabo actividades de laboratorio sin tener que cambiar de aula. Además, ofrecen alternativas para la enseñanza híbrida y a distancia.

El impacto de los laboratorios virtuales, y la disyuntiva entre experimentos tradicionales y virtuales, han sido objeto de un considerable interés por parte de la comunidad educativa en los últimos años en diferentes áreas de conocimiento: Física (Deutsch, 2014; Finkelstein, 2005; Finkelstein, 2006; Franco, 2013; Perkins, 2006; Wieman, 2008), Química (Bortnik, 2017; Climent-Bellido, 2003; García Armada, 2016; Jensen, 2013; Moore, 2014; Tatli, 2010), Biología y Ciencias de la Vida (Lewis, 2014; Sanz, 2005; Stuckey-Mickell, 2007) e Ingeniería (Chan, 2009; Heradio, 2016; Ma, 2006; Potkonjak, 2016).

En general, diferentes estudios, tanto de Ciencias Experimentales como de Ciencias de la Salud, muestran niveles similares de éxito con ambos enfoques en cuanto a calificaciones, adquisición de contenidos y/o valoración (Cobb, 2009; Darrah, 2014; De Jong, 2013; Hawkins, 2013; Klar, 2007; Lewis, 2014; Makransky, 2016; Pyatt, 2012; Spernjak, 2017; Triona, 2003; Tsihouridis, 2013; Winkelmann, 2014; Winkelmann, 2017), aunque recientes estudios comparativos sobre la eficacia de laboratorios tradicionales (LT) y laboratorios virtuales (LV) muestran que con los segundos los estudiantes adquieren el mismo nivel de aprendizaje, e incluso superior (Brinson, 2015; Faour, 2018; Herga, 2012; Martínez- 
Jiménez, 2003; Tatli, 2010; Toth, 2014; Zacharia, 2007), y una valoración general muy positiva (Bozkurt, 2010; McKagan, 2008; Olakanmi, 2015; Pyatt, 2012; Stuckey-Mickell, 2007; Tatli, 2010) en relación con diferentes variables (conocimiento y comprensión de contenidos, razonamiento científico, habilidades prácticas experimentales, interés y apreciación por la Ciencia, habilidades analíticas de investigación, manejo de datos, comunicación científica y trabajo en equipo, visualización de fenómenos microscópicos, etc.). De hecho, Merchant (2014) señala la gran utilidad didáctica de simulaciones, juegos y mundos virtuales. También se destaca el hecho de poder realizar prácticas de laboratorio muy complicadas o que requerirían medios muy costosos (Heradio, 2016; Lewis, 2014; Muhamad, 2010). Asimismo, diferentes estudios señalan que el alumnado que llevó a cabo simulaciones por ordenador adquirió un mayor dominio de los contenidos conceptuales en Física e incluso desarrolló una mayor destreza en la manipulación de componentes reales (Finkelstein, 2006; Tarekegn, 2009).

Por otro lado, también hay estudios que apuntan a que el aprendizaje en ciertas áreas como Química, Biología y Farmacología es más productivo con LT (es decir, las calificaciones son superiores o se recuerdan mejor los detalles experimentales) y/o más apreciado por el alumnado, mientras que en Física los resultados son similares con LT y LV (Brinson, 2015; Colorado DOHE, 2012; Hughes, 2001; Quinn, 2009). Sin embargo, estudiantes que realizaron experimentos tradicionales y virtuales análogos valoraron más positivamente, en ocasiones, la experiencia tradicional (Engum, 2003; Chan, 2009; Spernjak, 2017), lo que unido a las ventajas intrínsecas de la experiencia virtual debería conducir a la sinergia más que al debate en la comunidad educativa.

En este sentido, tal y como han señalado diferentes autores (Bortnik, 2017; Brinson, 2015; Cheesman, 2014; Chiu, 2015; Climent-Bellido, 2003; De Jong, 2013; Engum, 2003; Karpen, 2014; Kocijancic, 2004; Limniou, 2007; Liu, 2006; Makransky, 2016; Tsihouridis, 2014; Tsihouridis, 2015; Tsihouridis, 2017; Winkelmann, 2017; Zacharia, 2007; Zacharia, 2008), la clave para un aprendizaje significativo puede estar en la combinación de ambos tipos de experiencias: tradicionales y virtuales. En materias como Física y Química puede resultar muy útil que los estudiantes se percaten de las semejanzas y diferencias entre ambos enfoques en lo que respecta a resultados y a protocolos de trabajo, y que simultáneamente se beneficien de la combinación sinérgica de ambos tipos de experimentos:

$\checkmark \quad$ Los laboratorios tradicionales acercan al estudiante al trabajo de un científico, pero los laboratorios virtuales son más adecuados para discentes inexpertos porque, al no existir error experimental, los resultados son siempre los esperados de acuerdo con la teoría vista en clase.

$\checkmark \quad$ Los laboratorios tradicionales permiten mejorar la destreza de trabajo en equipo en un período corto de tiempo (la sesión de laboratorio), mientras que los laboratorios virtuales permiten la colaboración en línea entre los diferentes miembros del equipo durante un tiempo indefinido.

$\checkmark \quad$ Los laboratorios tradicionales permiten concienciar al alumnado de la importancia de la optimización del tiempo, del esfuerzo y de los recursos, mientras que los

(cc) EY-NC-ND 2018, Universitat Politècnica de València

Congreso IN-RED (2018) 
laboratorios virtuales permiten repetir las experiencias siempre que sea necesario y a un ritmo adecuado para cada estudiante.

$\checkmark$ Se pone de manifiesto la importancia del error experimental en experimentos tradicionales al comparar los resultados obtenidos con la práctica virtual, fomentando así la reflexión acerca de la diferencia entre situaciones ideales (a través del LV) y situaciones reales (a través del LT).

$\checkmark$ Muchos laboratorios virtuales están diseñados en modo "abierto” y flexible, de modo que el usuario puedes utilizarlos de formas muy diferentes, realizando experiencias "a la carta”, lo que puede servirle para alcanzar un amplio abanico de objetivos didácticos.

\section{Objetivos}

El principal objetivo de este estudio es analizar el impacto de los laboratorios virtuales y tradicionales en asignaturas de Ciencias Básicas (CCBB) en un grado de Ciencias de la Salud.

En este trabajo nos hemos centrado en la percepción del alumnado como herramienta fundamental para distinguir la experiencia de los discentes con ambas aproximaciones didácticas. Esto puede ser crucial, puesto que, en general, los estudios que muestran un mayor éxito de los LT ponen más énfasis en los contenidos y la comprensión conceptual (por lo que utilizan exámenes y pruebas de conocimiento como herramientas de evaluación), mientras que los estudios que inclinan la balanza en favor de los LV se apoyan preferentemente en datos cualitativos relacionados con la percepción del profesorado y/o del alumnado (Brinson, 2015). Sin embargo, hemos de considerar que encontramos muchas excepciones en la literatura científica, y además no son iguales todas las asignaturas de Ciencias ni tampoco son iguales los diferentes perfiles de estudiantes que estudian uno u otro grado.

Para evaluar la percepción del alumnado con respecto a ambos tipos de laboratorios hemos analizado cinco variables: valoración general, grado de satisfacción, aumento de motivación y aumento del rendimiento, investigándose también las posibles diferencias de género.

\section{Desarrollo de la innovación}

Los participantes del estudio fueron 129 estudiantes del grado en Odontología $\left(n_{\text {hombres }}=59\right.$; $\left.n_{\text {mujeres }}=70\right)$ entre 18 y 46 años $(M=20.73 ; D T=0.72)$ de una universidad española. Todos los participantes firmaron un consentimiento informado antes de su participación en el estudio. Los estudiantes universitarios que participaron en el estudio estaban distribuidos en grupos diferentes, algunos de los cuales recibieron docencia en español, y otros en inglés. Los estudiantes procedían de 20 países, principalmente de Italia (50.4\%), Francia (17.1\%), Alemania (7\%) y España (6.2\%), aunque también de Reino Unido (3.9\%) y Marruecos (2.3\%). La media de edad fue de $20.73 \pm 3.09$ años (rango 18-46), con un $54.3 \%$ de mujeres 
y un $45.7 \%$ de varones. A lo largo del curso académico los alumnos recibieron diversas prácticas equivalentes en formato tradicional y en formato virtual en dos asignaturas de CCBB: bioquímica y microbiología.

El enfoque de este estudio es longitudinal y cuantitativo. Al principio del curso los estudiantes respondieron a un primer cuestionario con diferentes ítems para valorar tanto sus conocimientos previos (las asignaturas científicas que habían estudiado en la etapa preuniversitaria, si estaban acostumbrados a trabajar con ordenadores, de qué asignaturas habían hecho prácticas de laboratorio y a qué nivel creían dominar las materias de CCBB pre-universitarias) como su apreciación e interés hacia las CCBB, tanto de forma individual como el conjunto de las mismas (importancia para su grado, importancia para su profesión, motivación...). Asimismo, también se analizó su implicación académica y los diferentes tipos de motivación al cursar sus estudios actuales. Dicho cuestionario estaba compuesto mayoritariamente por ítems que se podían valorar mediante una escala de Likert. Análogamente, al final del curso académico los alumnos completaron un paquete de cuestionarios para evaluar la valoración y el impacto de ambos enfoques (LT y LV), por un lado, y también para analizar la variación en los ítems anteriores que aparecían en el primer cuestionario.

Este análisis está en progreso en la actualidad, por lo que en el presente estudio abordaremos únicamente la valoración de los diferentes tipos de prácticas de laboratorio una vez terminado el curso académico. El análisis de datos (Greasley, 2008; Muijs, 2014; Pardo, 2015) se llevó a cabo con el programa SPSS (IBM Corp. Released 2013. IBM SPSS Statistics for Windows, Version 22.0. Armonk, NY: IBM Corp).

\section{Resultados}

En este estudio piloto nos hemos centrado en una serie de variables para evaluar la apreciación de los estudiantes hacia ambos tipos de experimentos: LT y LV. La valoración general de las prácticas se midió analizando la media de las respuestas de una serie de adjetivos positivos (i.e. "interesantes”, “útiles”, “divertidas”, “fáciles” y "curiosas”) valorados a través de una escala de Likert de 1 (muy bajo) a 5 (muy alto). La satisfacción con la realización de las prácticas se evaluó a través de un único ítem, mientras que el aumento de la motivación hacia las CCBB y el aumento del rendimiento en las asignaturas de $C C B B$ resultan de la media aritmética de las apreciaciones del alumnado (evaluadas a través de una escala tipo Likert desde 1 muy bajo a 5 muy alto) de las diferentes asignaturas cursadas.

Como puede observarse en la Figura 1, los alumnos que participaron en este estudio indicaron que estaban más satisfechos con los laboratorios tradicionales que con los virtuales. Estos resultados se observan tanto al analizar la muestra conjuntamente como al analizar la muestra de hombres y mujeres por separado. Asimismo, los resultados indican que existen diferencias significativas en la satisfacción de los universitarios a favor de los laboratorios tradicionales $(t=-6.937 ;$ g.l. $=112 ; p=.000)$.

(cc) BY-NC-ND 2018, Universitat Politècnica de València

Congreso IN-RED (2018) 


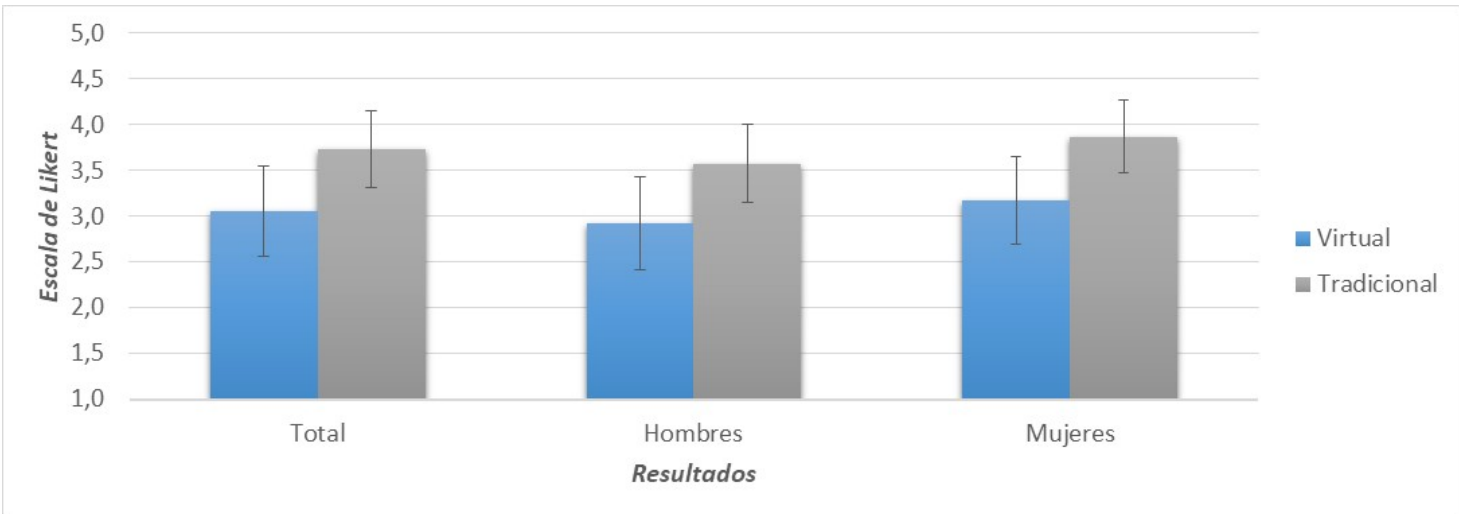

Fig. 1. Satisfacción del alumnado con las prácticas virtuales y tradicionales.

En la misma línea, los resultados del estudio muestran que los universitarios valoraban más positivamente los laboratorios tradicionales que los laboratorios virtuales realizados en las asignaturas de CCBB (véase Figura 2). Al comparar la media de ambos tipos de prácticas, los resultados indican que existen diferencias significativas en la valoración general de las prácticas a favor de los laboratorios tradicionales $(t=-6.981$; g.l. $=127 ; p=.000)$.

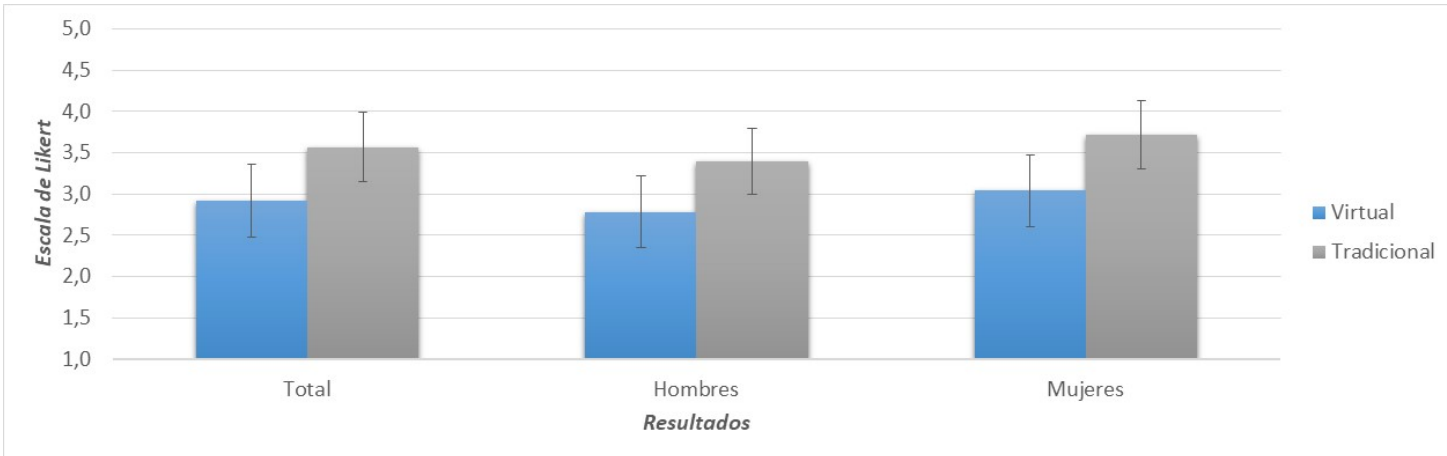

Fig. 2. Valoración positiva del alumnado de las prácticas virtuales y tradicionales.

En relación al rendimiento del alumnado en las asignaturas de CCBB, los resultados del estudio indican que los participantes percibían que su rendimiento aumentaba más a través de la realización de prácticas tradicionales (véase Figura 3). Estos resultados mantenían la misma tendencia tanto analizando las respuestas de hombres y mujeres por separado como evaluando sus respuestas conjuntamente. Al analizar la existencia de diferencias significativas, los resultados confirman la existencia de dichas diferencias $(t=-6.228$; g.l. $=128 ; p=.000$ ) a favor de las prácticas tradicionales. 


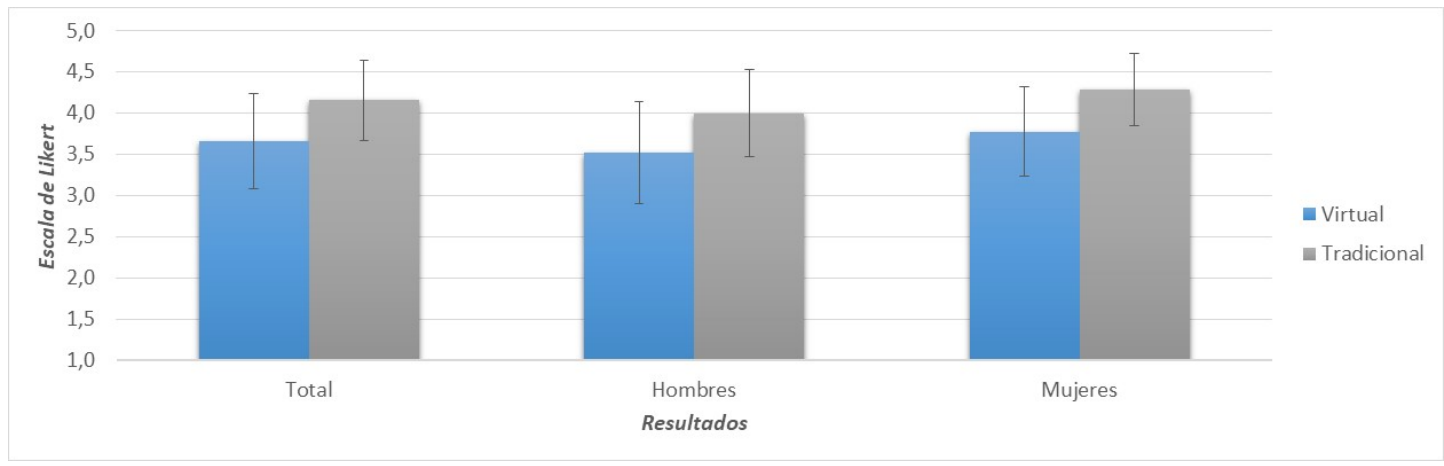

Fig. 3. Aumento del rendimiento en las asignaturas de CCBB tras la realización de prácticas virtuales y tradicionales

Por otro lado, en relación a la motivación de los universitarios respecto a las asignaturas de CCBB, en la Figura 4 se puede observar que los participantes del presente estudio indicaron que su motivación hacia las asignaturas de CCBB aumentaba más tras la realización de prácticas tradicionales. Estas diferencias resultaron ser significativas $(t=-5.016 ;$ g.l. $=127$; $p=.000)$.

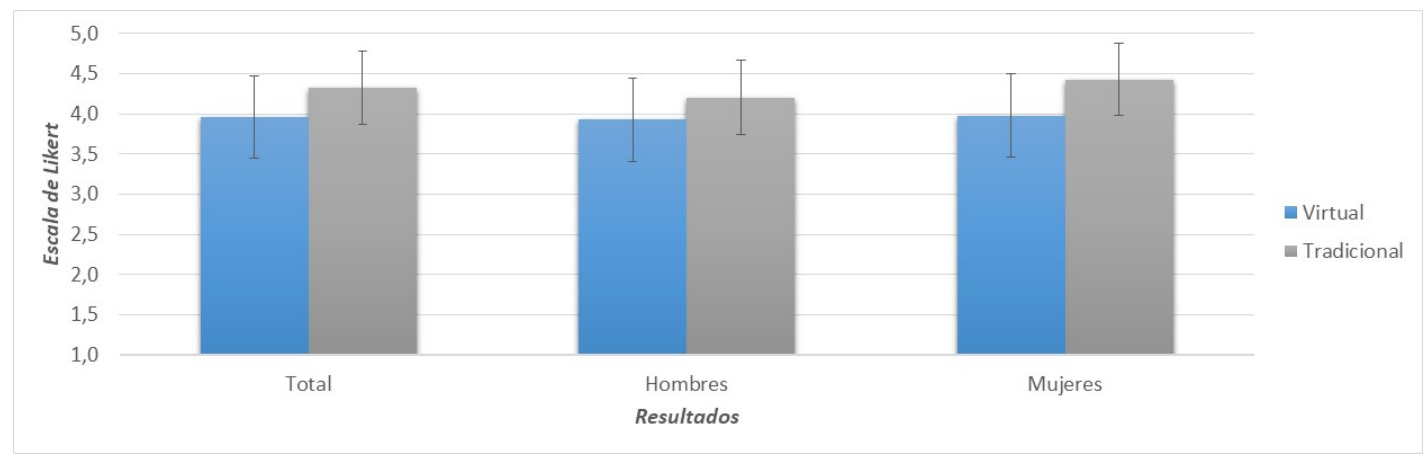

Fig. 4. Aumento de la motivación en las asignaturas de CCBB tras la realización de prácticas virtuales y tradicionales.

En relación con las diferencias de género, los resultados indicaron que las mujeres valoraban significativamente mejor las prácticas tradicionales $(t=-2.227$; g.l. $=124 ; p=$ .028), y referían una mayor satisfacción general con este tipo de prácticas $(t=-2.027 ;$ g.l. $=$ $127 ; p=.045)$.

Además, es importante destacar que tanto los LT como los LV condujeron a un aumento de la motivación hacia las CCBB y a una mejor valoración de las mismas, así como una mejora del rendimiento académico, siempre desde la perspectiva de apreciación de los discentes. En la Tabla 1 pueden observarse las medias y las desviaciones típicas de la percepción de aumento de motivación y percepción de mejora del rendimiento en las asignaturas de CCBB a través de la realización de ambos tipos de prácticas. Estos

(cc) EY-Nc-No 2018, Universitat Politècnica de València

Congreso IN-RED (2018) 
resultados muestran una mayor percepción en el aumento de la motivación y del rendimiento en todas las asignaturas a través de la realización de los laboratorios tradicionales.

Tabla 1. Resultados de valoración de las experiencias con LT y LV en diferentes asignaturas.

\begin{tabular}{lccccc}
\hline Materia & Rango & $\begin{array}{c}\text { Aumento de } \\
\text { motivación } \\
\text { con LV }\end{array}$ & $\begin{array}{c}\text { Aumento de } \\
\text { motivación } \\
\text { con LT }\end{array}$ & $\begin{array}{c}\text { Mejora de } \\
\text { rendimiento } \\
\text { con LV }\end{array}$ & $\begin{array}{c}\text { Mejora de } \\
\text { rendimiento } \\
\text { con LT }\end{array}$ \\
\hline Bioquímica & $1-5$ & $3.80(1.27)$ & $4.14(1.23)$ & $3.71(1.34)$ & $4.09(1.21)$ \\
Microbiología & $1-5$ & $4.43(1.06)$ & $4.57(1.00)$ & $3.98(1.17)$ & $4.43(1.01)$ \\
\hline
\end{tabular}

En lo referente a los adjetivos positivos, y en concordancia con los resultados anteriores, los LT siempre son mejor valorados que los LV, tal y como se puede observar en la Tabla 2:

Tabla 2. Valoración positiva de las prácticas virtuales y tradicionales.

\begin{tabular}{lccc}
\hline \multicolumn{1}{c}{ Adjetivos } & Rango & $\begin{array}{c}\text { LV } \\
\text { M (DT) }\end{array}$ & $\begin{array}{c}\text { LT } \\
\text { (DT) }\end{array}$ \\
\hline Interesantes & & $3.02(0.99)$ & $3.78(0.94)$ \\
Útiles & $1-5$ & $2.96(1.10)$ & $3.75(0.88)$ \\
Divertidas & $1-5$ & $2.61(1.14)$ & $3.44(1.11)$ \\
Fáciles & $1-5$ & $3.01(1.06)$ & $3.18(0.92)$ \\
Curiosas & $1-5$ & $3.02(1.11)$ & $3.68(1.03)$ \\
\hline
\end{tabular}

Por otro lado, tal y como se puede ver en la Figura 5, el alumnado prefiere hacer preferentemente LT (49.6\%) o incluso hacer solo LT (21.7\%) frente a hacer el mismo número de LT y de LV (14.7\%), hacer preferentemente LV (12.4\%) o hacer solo LV (0.8\%). Este resultado está en la línea de los hallazgos de Engum (2003), Chan (2009), Quinn (2009) o Stuckey-Mickell (2007), aunque difiere de otros estudios en los que se valoraban de forma similar ambos tipos de prácticas (Lewis, 2014; Tatli, 2010; Winkelmann, 2014; Faour, 2018), o en los que se apuntaba a una mejor valoración de los LV (Bozkurt, 2010; Pyatt, 2012). En este sentido, hemos de tener en cuenta el perfil del alumnado: por un lado, se trata de personas acostumbradas a trabajar y a divertirse con herramientas digitales, por lo que el LT puede suponer una novedad respecto al LV; por otro lado, son estudiantes de grados de Ciencias de la Salud en cuyo futuro trabajo los 
contenidos procedimentales serán cruciales. Este interés por mejorar sus destrezas podría motivarles más, de entrada, hacia los LT en CCBB.

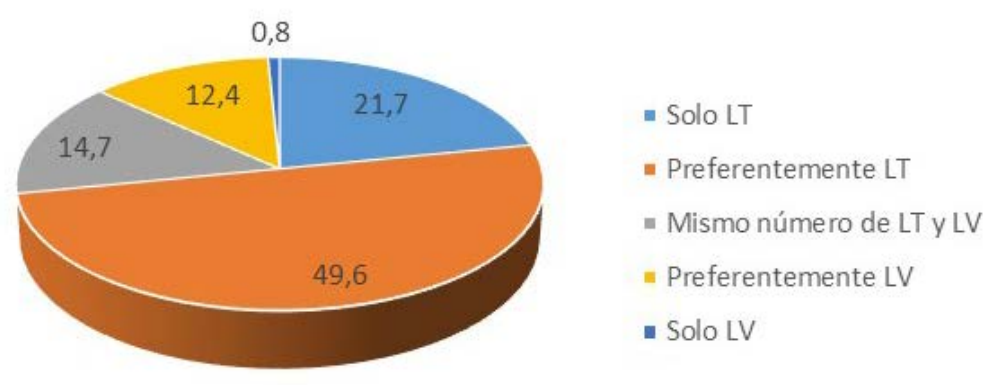

Fig. 5. Preferencias del alumnado en cuanto a la realización de experimentos virtuales y experimentos virtuales durante el curso.

\section{Conclusiones}

Los resultados obtenidos muestran que los estudiantes prefieren las prácticas tradicionales a las virtuales en todos los aspectos: valoración general, grado de satisfacción, aumento de motivación y aumento del rendimiento. Estos resultados resaltan la importancia de mantener un número considerable de prácticas tradicionales en los grados en Ciencias de la Salud para potenciar la motivación y el rendimiento del alumnado en las asignaturas de CCBB. Esto es importante puesto que son asignaturas que los discentes tienden a percibir como alejadas de su futura práctica profesional, por lo que su motivación suele ser inferior a la de otras asignaturas del grado.

Sin embargo, el rol de los laboratorios virtuales en la educación del presente y del futuro no debe subestimarse. En este sentido, la clave es conseguir que el estudiante no se muestre pasivo en el aula, sino que participe y actúe, e incluso que explore y descubra cosas motu proprio, objetivo que se puede conseguir más fácilmente con aplicaciones virtuales on-line.

En futuros trabajos consideraremos los diferentes tipos de motivación del alumnado, su apreciación de la importancia de las ciencias básicas para su grado y para su futuro profesional, así como los conocimientos previos respecto a actividades de laboratorio y a conocimientos informáticos. Asimismo, se está valorando la opción de estudiar cualitativamente las apreciaciones del alumnado mediante entrevistas aleatorias con la finalidad de llevar a cabo una triangulación de los datos obtenidos. Por último, también se intentará contar con una muestra $n$ de mayor tamaño, teniendo en cuenta además a estudiantes de diferentes grados de Ciencias de la Salud (no solo de Odontología, sino también de Fisioterapia y de Enfermería).

(c) EY-NC-ND 2018, Universitat Politècnica de València

Congreso IN-RED (2018) 


\section{Referencias}

AMERICAN CHEMICAL SOCIETY. ACS position statement: Importance of hands-on laboratory

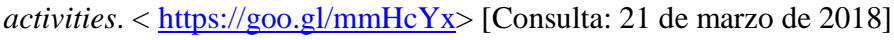

BORTNIK, B., STOZHKO, N., PERVUKHINA, I., TCHERNYSHEVA, A. y BELYSHEVA, G. (2017). "Effect of virtual analytical chemistry laboratory on enhancing student research skills and practices" en Research in Learning Technology Research in Learning Technology, 25, 1-20.

BOZKURT, E. e ILIK, A. (2010). “The effect of computer simulations over students' beliefs on physics and physics success” en Procedia - Social and Behavioral Sciences, 2, 4587-4591.

BRINSON, J. R. (2015). "Learning outcome achievement in non-traditional (virtual and remote) versus traditional (hands-on) laboratories: A review of the empirical research” en Computers \& Education, 87, Supplement C, 218-237.

CHAN, C., y FOK, W. (2009). "Evaluating learning experiences in virtual laboratory training through student perceptions: a case study in Electrical and Electronic Engineering at the University of Hong Kong” en Engineering Education, 4, 2, 70-75.

CHEESMAN, M.J., CHEN, S., MANCHADI, M.-L., JACOB, T., MINCHIN, R.F. y TREGLOAN, P.A. (2014). "Implementation of a Virtual Laboratory Practical Class (VLPC) module in pharmacology education” en Pharmacognosy Communication, 4, 2-10.

CHIU, J. L., DEJAEGHER, C. J. y CHAO, J. (2015). “The effects of augmented virtual science laboratories on middle school students' understanding of gas properties” en Computers \& Education, 85, 59-73.

CLIMENT-BELLIDO, M. S., MARTÍNEZ-JIMÉNEZ, P., PONTES-PEDRAJAS, A., y POLO, J. (2003). "Learning in Chemistry with Virtual Laboratories" en Journal of Chemical Education, 80, 3, 346352.

COBB, S., HEANEY, R., CORCORAN, O. y HENDERSON-BEGG, S. (2009). "The Learning Gains and Student Perceptions of a Second Life Virtual Lab” en Bioscience Education, 13, 1-9.

COLL, C., MARTÍN, E., MAURI, T., MIRAS, M., ONRUBIA, J., SOLÉ, I., et al. (2007). El constructivismo en el aula (18 ${ }^{\mathrm{a}}$ ed.). Barcelona: Editorial Graó.

COLORADO DOHE (Department of Higher Education, 2012). “Online versus traditional learning: A comparison study of Colorado community college science classes”. <https://goo.gl/E3j2Yf> [Consulta: 21 de marzo de 2018].

COOK, E., KENNEDY, E., y MCGUIRE, S. Y. (2013). "Effect of Teaching Metacognitive Learning Strategies on Performance in General Chemistry Courses” en Journal of Chemical Education, 90, 8, 961-967.

DARRAH, M., HUMBERT, R., FINSTEIN, J., SIMON, M. y HOPKINS, J. (2014). “Are Virtual Labs as Effective as Hands-on Labs for Undergraduate Physics? A Comparative Study at Two Major Universities” en Journal of Science Education and Technology, 23, 803-814.

DE JONG, T., LINN, M. C. y ZACHARIA, Z. C. (2013). "Physical and Virtual Laboratories in Science and Engineering Education” en Science, 340, 305-308.

DEUTSCH, J. M. (2014). “Biophysics software for interdisciplinary education and research” en American Journal of Physics, 82, 5, 442-450.

(c) 2018, Universitat Politècnica de València

Congreso In-Red (2018) 
DOMIN, D. S. (1999). “A Review of Laboratory Instruction Styles” en Journal of Chemical Education, 76, 4, 543.

ELLIOTT, M. J., STEWART, K. K. y LAGOWSKI, J. J. (2008). “The Role of the Laboratory in Chemistry Instruction” en Journal of Chemical Education, 85, 145-149.

ENGUM, S. A., JEFFRIES, P., y FISHER, L. (2003). “Intravenous catheter training system: Computerbased education versus traditional learning methods” en The American Journal of Surgery, 186, 1, 6774.

FAOUR, M. A. y AYOUBI, Z. (2018). "The Effect of Using Virtual Laboratory on Grade 10 Students' Conceptual Understanding and their Attitudes towards Physics” en Journal of Education in Science, Environement and Health, 4, 54-68.

FINKELSTEIN, N. D., ADAMS, W. K., KELLER, C. J., KOHL, P. B., PERKINS, K. K., PODOLEFSKY, N. S., et al. (2005). "When learning about the real world is better done virtually: A study of substituting computer simulations for laboratory equipment” en Physical Review Special Topics Physics Education Research, 1, 1, 010103.

FINKELSTEIN, N. D., ADAMS, W. K., PERKINS, K. K., y WIEMAN, C. (2006). "HighTech Tools for Teaching Physics: the Physics Education Technology Project” en Journal of Online Learning and Teaching, 2, 3, 110-121.

FRANCO, A., BELÉNDEZ, A., y ABLANQUE, J. (2013). "Recursos multimedia para la enseñanza on-line de la Física” en Revista Española de Física, 27, 1, 49-56.

FREEMAN, S., EDDY, S. L., MCDONOUGH, M., SMITH, M. K., OKOROAFOR, N., JORDT, H., et al. (2014). “Active learning increases student performance in science, engineering, and mathematics” en Proceedings of the National Academy of Sciences, 111, 23, 8410-8415.

GARCIA ARMADA, M. P., y FERNANDEZ-AVILES PEDRAZA, D. (2016). "Prácticas de Química Orgánica posibles gracias a las nuevas tecnologías” en M. González, A. Baratas y A. Brandi (Eds.). IV Congreso de Docentes de Ciencias (pp. 229-238). Madrid: Santillana.

GREASLEY, P. (2008). Quantitative Data Analysis Using SPSS: An Introduction for Health \& Social Science. New York: McGraw-Hill Open University Press.

HAWKINS, I. \& PHELPS, A. J. (2013). "Virtual laboratory vs. traditional laboratory: which is more effective for teaching electrochemistry?” en Chem. Educ. Res. Pract., 14, 516-523.

HERADIO, R., DE LA TORRE, L., GALAN, D., CABRERIZO, F. J., HERRERA-VIEDMA, E., y DORMIDO, S. (2016). "Virtual and remote labs in education: A bibliometric analysis"en Computers \& Education, 98, Supplement C, 14-38.

HERGA, N. y DINEVSKI, D. (2012). "Virtual Laboratory in Chemistry - Experimental Study of Understanding, Reproduction and Application of Acquired Knowledge of Subject's Chemical Content” en Organizacija, 45, 3, 108-116.

HERREID, C. F. (2012). "ConfChem Conference on Case-Based Studies in Chemical Education: The Future of Case Study Teaching in Science” en Journal of Chemical Education, 90, 2, 256-257.

HOFSTEIN, A., y LUNETTA, V. N. (2004). “The laboratory in science education: Foundations for the twenty-first century” en Science Education, 88, 1, 28-54.

HYSLOP-MARGISON, E. J., y STROBEL, J. (2007). “Constructivism and education: misunderstandings and pedagogical implications” en The Teacher Educator, 43, 1, 72-86.

(cc) EY-NC-ND 2018, Universitat Politècnica de València

Congreso IN-RED (2018) 
HUGHES, I. E. (2001). “Do computer simulations of laboratory practicals meet learning needs?” en Trends in Pharmacological Sciences, 22, 71-74.

JENSEN, J. H., y KROMANN, J. C. (2013). “The Molecule Calculator: A Web Application for Fast Quantum Mechanics-Based Estimation of Molecular Properties” en Journal of Chemical Education, 90, 8, 1093-1095.

KARPEN, M. E., HENDERLEITER, J., y SCHAERTEL, S. A. (2004). "Integrating Computational Chemistry into the Physical Chemistry Laboratory Curriculum: A Wet Lab/Dry Lab Approach” en Journal of Chemical Education, 81, 4, 475-477.

KLAR, D., TRIONA, L.M. y CAMERON, W. (2007). "Hands on what? The relative effectiveness of physical versus virtual materials in an engineering design project by middle school children” en Journal of Research in Science Teaching, 44, 183-203.

KRATHWOHL, D. R. (2002). “A Revision of Bloom's Taxonomy: An Overview” en Theory Into Practice, $41,4,212-264$.

KOCIJANCIC, S., y O'SULLIVAN, C. (2004). "Real or Virtual Laboratories in Science Teaching -is this Actually a Dilemma? ” en Informatics in Education, 3, 2, 239-250.

LEWIS, D. I. (2014). “The pedagogical benefits and pitfalls of virtual tools for teaching and learning laboratory practices in the biological sciences”. Higher Education Academy, Vol. 4, pp. 22-30.

LIMNIOU, M., PAPADOPOULOS, N., GIANNAKOUDAKIS, A., ROBERTS, D. \& OTTO, O. (2007). "The integration of a viscosity simulator in a chemistry laboratory" en Chemistry Education Research and Practice, 8, 220-231.

LIU, X. (2006). "Effects of Combined Hands-on Laboratory and Computer Modeling on Student Learning of Gas Laws: A Quasi-Experimental Study” en Journal of Science Education and Technology, 15, 1, 89-100.

LIVINGSTONE, S. (2012). "Critical reflections on the benefits of ICT in education”. Oxford Review of Education, Vol. 38(1), pp. 9-24.

MA, J., y NICKERSON, J. V. (2006). "Hands-on, simulated, and remote laboratories: A comparative literature review” en ACM Computing Surveys, 38, 3.

MAKRANSKY, G., THISGAARD, M. W. y GADEGAARD, H. (2016). "Virtual simulations as preparation for lab exercises: Assessing learning of key laboratory skills in microbiology and improvement of essential non-cognitive skills” en Plos One, 11, 1-11.

MAZUR, E. (2009). “Farewell, Lecture? ” en Science, 323, 5910, 50-51.

MERCHANT, Z., GOETZ, E. T., CIFUENTES, L., KEENEY-KENNICUTT, W. y DAVIS, T. J. (2014). "Effectiveness of virtual reality-based instruction on students' learning outcomes in K-12 and higher education: A meta-analysis” en Computers \& Education, 70, 29-40.

MCKAGAN, S. B., PERKINS, K. K., DUBSON, M., MALLEY, C., REID, S., LEMASTER, R., et al. (2008). "Developing and researching PhET simulations for teaching quantum mechanics" en American Journal of Physics, 76, 4, 406-417.

MICHAEL, J. (2006). "Where's the evidence that active learning works? ” en Advances in Physiology Education, 30, 4, 159-167.

MOORE, E. B., CHAMBERLAIN, J. M., PARSON, R., y PERKINS, K. K. (2014). "PhET Interactive Simulations: Transformative Tools for Teaching Chemistry” en Journal of Chemical Education, 91, 8, 1191-1197. 
MUHAMAD, M., ZAMAN, H. B., Y AHMAD, A. (2010). "Virtual laboratory for learning biology - a preliminary investigation” en World Academy of Science, Engineering and Technology, 71, 572-575.

MUIJS, D. (2004). Doing quantitative research in education with SPSS. London: SAGE Publications Ltd.

NATIONAL SCIENCE TEACHERS ASSOCIATION. NSTA position statement: The integral role of laboratory investigations in science instruction. < https://goo.gl/CgL67b $>$ [Consulta: 21 de marzo de 2018].

OLAKANMI, E. E. (2015). "The Effects of a Web-Based Computer Simulation on Students' Conceptual Understading of rate of reaction and attitude towards chemistry"en Journal of Baltic Science Education, 14(5). 627-641.

PARDO, A., RUIZ, M. Á., y SAN MARTÍN, R. (2015). Análisis de datos en ciencias sociales y de la salud I (2a ed.). Madrid: Editorial Síntesis.

PERKINS, K., ADAMS, W., DUBSON, M., FINKELSTEIN, N., REID, S., WIEMAN, C., et al. (2006). "PhET: Interactive Simulations for Teaching and Learning Physics" en The Physics Teacher, 44, 1823.

PHILLIPS, D. C. (1995). “The Good, the Bad, and the Ugly: The Many Faces of Constructivism” en Educational Researcher, 24, 7, 5-12.

PINTO, G., ESCUDERO, P., y MARTIN, M. (2008). "Aportaciones sobre el aprendizaje activo de la química” en Anales de Química, 104, 3, 211-214.

POTKONJAK, V., GARDNER, M., CALlAGHAN, V., MATTILA, P., GUETL, C., PETROVIC, V. M., et al. (2016). "Virtual laboratories for education in science, technology, and engineering: A review" en Computers \& Education, 95, Supplement C, 309-327.

POZO, J. I., y GOMEZ, M. A. (2006). Aprender y enseñar ciencia: del conocimiento cotidiano al conocimiento científico ( $7^{\mathrm{a}}$ ed.). Madrid: Ediciones Morata.

PRINCE, M. (2004). “Does Active Learning Work? A Review of the Research” en Journal of Engineering Education, 93, 3, 223-231.

PYATT, K. y SIMS, R. (2012). "Virtual and Physical Experimentation in Inquiry-Based Science Labs: Attitudes, Performance and Access” en Journal of Science Education and Technology, 21, 133-147.

ROCARD, M., CSERMELY, P., JORDE, D., LENZEN, D., WALBERG-HENRIKSSON, H., y HEMMO, V. (2007). "Science education now: a renewed pedagogy for the future of Europe". Brussels: European Commission: Directorate-General for Research.< https://goo.gl/vhVrvv> [Consulta: $21 \mathrm{de}$ marzo de 2018].

SANMARTI, N. (2009). Didáctica de las ciencias en la educación secundaria obligatoria (7ª ed.). Madrid: Editorial Síntesis.

SANZ, A., Y MARTINEZ, J. (2005). "El uso de los laboratorios virtuales en la asignatura Bioquímica como alternativa para la aplicación de las tecnologías de la información y la comunicación” en Tecnología Química, 25, 1, 5-17.

SMAGORINSKY, P. (1995). "The Social Construction of Data: Methodological Problems of Investigating Learning in the Zone of Proximal Development” en Review of Educational Research, 65, 3, 191-212.

SPENCER, J. N. (1999). "New Directions in Teaching Chemistry: A Philosophical and Pedagogical Basis" en Journal of Chemical Education, 76(4), 566-569.

(cc) EY-NC-ND 2018, Universitat Politècnica de València

Congreso IN-RED (2018) 
ŠPERNJAK, A. y ŠORGO, A. (2018). "Differences in acquired knowledge and attitudes achieved with traditional, computer-supported and virtual laboratory biology laboratory exercises” en Journal of Biological Education, 52, 206-220.

SPRAWLS, P. (2008). "Evolving models for medical physics education and training: a global perspective" en Biomedical imaging and intervention journal, 4, 1, e16-e16.

STUCKEY-MICKELL, T. A., y STUCKEY-DANNER, B. D. (2007). "Virtual Labs in the Online Biology Course: Student Perceptions of Effectiveness and Usability” en Journal of Online Learning and Teaching, 3, 2, 105-111.

TAREKEGN, G. (2009). “Can computer simulations substitute real laboratory apparatus?” en LatinAmerican Journal of Physics Education, 3, 3, 506-517.

TOTH, E. E., LUDVICO, L. R. y MORROW, B. L. (2014). "Blended inquiry with hands-on and virtual laboratories: the role of perceptual features during knowledge construction” en Interactive Learning Environments, 22, 614-630.

TRIONA, L. M., y KLAHR, D. (2003). Point and Click or Grab and Heft: Comparing the Influence of Physical and Virtual Instructional Materials on Elementary School Students' Ability to Design Experiments en Cognition and Instruction, 21(2), 149-173.

TSIHOURIDIS, C., VAVOUGIOS, D. y IOANNIDIS, G. S. (2013). “The effectiveness of virtual laboratories as a contemporary teaching tool in the teaching of electric circuits in Upper High School as compared to that of real labs" en 2013 International Conference on Interactive Collaborative Learning (ICL), 25-27 Sept. 2013, Kazan, 816-820.

TSIHOURIDIS, C., VAVOugios, D., IOANnidis, G. S., AlEXIAS, A., ARGYROPOUlOS, C. y POULIOS, S. (2014). "Using sensors and data-loggers in an integrated mobile school-lab setting to teach Light and Optics” en 2014 International Conference on Interactive Collaborative Learning (ICL), 3-6 Dic. 2014, Dubai, 439-445.

TSIHOURIDIS, C., VAVOugios, D., IOANNIDIS, G. S., AlEXIAS, A., ARGYROPOUlOS, C. y POULIOS, S. (2015). "The effect of teaching electric circuits switching from real to virtual lab or vice versa; A case study with junior high-school learners” en 2015 International Conference on Interactive Collaborative Learning (ICL), 20-24 Sept. 2015, Florencia, 643-649.

TSIHOURIDIS, C. A., VAVOUGIOS, D. y IOANNIDIS, G. S. (2017). “The Effect of Switching the Order of Experimental Teaching in the Study of Simple Gravity Pendulum - Study with Junior High-school Learners” en International Journal of Emerging Technologies in Learning, 12, 128-141.

WIEMAN, C. E., PERKINS, K. K., y ADAMS, W. K. (2008). “Oersted Medal Lecture 2007: Interactive simulations for teaching physics: What works, what doesn't, and why” en American Journal of Physics, 76, 4, 393-399.

WINKELMANN, K., SCOTT, M. y WONG, D. (2014). “A Study of High School Students’ Performance of a Chemistry Experiment within the Virtual World of Second Life” en Journal of Chemical Education, 91, 1432-1438.

WINKELMANN, K., KEENEY-KENNICUTT, W., FOWLER, D. y MACIK, M. (2017). "Development, Implementation, and Assessment of General Chemistry Lab Experiments Performed in the Virtual World of Second Life” en Journal of Chemical Education, 94, 849-858.

ZACHARIA, Z.C. (2007). "Comparing and combining real and virtual experimentation: an effort to enhance students' conceptual understanding of electric circuits” en Journal of Computer Assisted Learning, 23, 120-132.

ZACHARIA, Z.C., OLYMPIOU, G.. y PAPAEVRIPIDOU, M. (2008). "Effects of experimenting with physical and virtual manipulatives on students' conceptual understanding in heat and temperature" en Journal of Research in Science Teaching, 45, 1021-1035.

(c) 2018, Universitat Politècnica de València

Congreso In-Red (2018) 